\title{
The Oecetis reticulata species-group from the South-West Pacific area (Trichoptera: Leptoceridae)
}

\author{
Arturs Neboiss \\ Department of Entomology, Museum of Victoria, 71 Victoria Crescent, Abbotsford, Vic. 3067, \\ Australia
}

Keywords: Trichoptera, Oecetis reticulata species-group, S.W. Pacific

\section{Summary}

Thirteen Oecetis species of the south-west Pacific area from Sulawesi, to Northern Australia and the Solomon Islands are placed in a new reticulata species-group on the basis of the distinctive shield-like tergite 8 in the males. Twelve new species are described in this paper: Oecetis accola sp. n., arawana sp. n., barbarae sp. n., catenulata sp. n., epekeina sp. n., kakaduensis sp. n., keraia sp. n., kolobota sp. n., oresbiosa sp. n. parmata sp. n., piptona sp. n. and xaniona sp. n.; and a thirteenth species, reticulata Kimmins, is figured for comparison.

\section{Résumé}

Treize espèces d'Oecetis de territoires du Pacifique du sud-ouest (Sulawesi, Australie septentrionale, Iles Solomon) sont placées dans le nouveau groupe d'espèces reticulata; les mâles de toutes ces espèces soont caractérisés sont décrites dans le présent travail: Oecetis accola sp. n., arawana sp. n., barbarae sp. n., catenulata sp. n., epekeina sp. n., kakaduensis sp. n., kereia sp. n., kolobata sp. n., oresbiosa sp. n., paramata sp. n., piptona $\mathrm{sp}$. $\mathrm{n}$. et xaniona $\mathrm{sp}$. $\mathrm{n}$.; une treizième espèce, reticulata Kimmins, est figurée pour permettre une comparaison.

\section{Introduction}

The cosmopolitan genus Oecetis McLachlan is represented in the south-west Pacific area by numerous species of which at least some, fall into more or less clearly recognisable groups. One species-group is distinguished by an unusual shieldlike abdominal tergite 8 in males (Fig. 2). The tergite is somewhat thickened, with internal honey- combed microstructure, and cuticle with fine, regularly reticulate surface pattern. The shield-like tergite 8 is extended distally and partially conceal the genitalic structures. In addition, small and somewhat angular or rounded patches of finely reticulate cuticle usually occur laterally on tergites 6 and 7 (Fig. 2).

Oecetis reticulata Kimmins, 1957 was the first species to be described from south-west Pacific area posessing the characteristic shield-like tergite 8 in the male. In this paper a further twelve new species are described and illustrated. The original figure of $O$. reticulata is reproduced for comparison (Fig. 32). Based on male genitalic features the reticulata species-group is established. Females of seven species are associated and their descriptions and illustrations included. They confirm closely to others in the genus.

Other Oecetis species with reticulate patches on distal abdominal tergites have been recorded from other zoogeographic regions. The reticulations were first noted and described by McLachlan (1877) in the European species Oecetis notata Rambur and Oecetis testacea Curtis, he referred to them as ' $a$ very sharply-defined group'. Detailed study of the structures in both species by Ris (1904, 1905), suggested that the reticulate areas probably function as scent organs. Two more species with such structures were recorded from North America by Betten (1934), O. avara (Banks) and O. resurgens (Walker) (=cinarescens Hagen), and subsequently, Ross (1944) referred to the reticulations in $O$. persimilis 


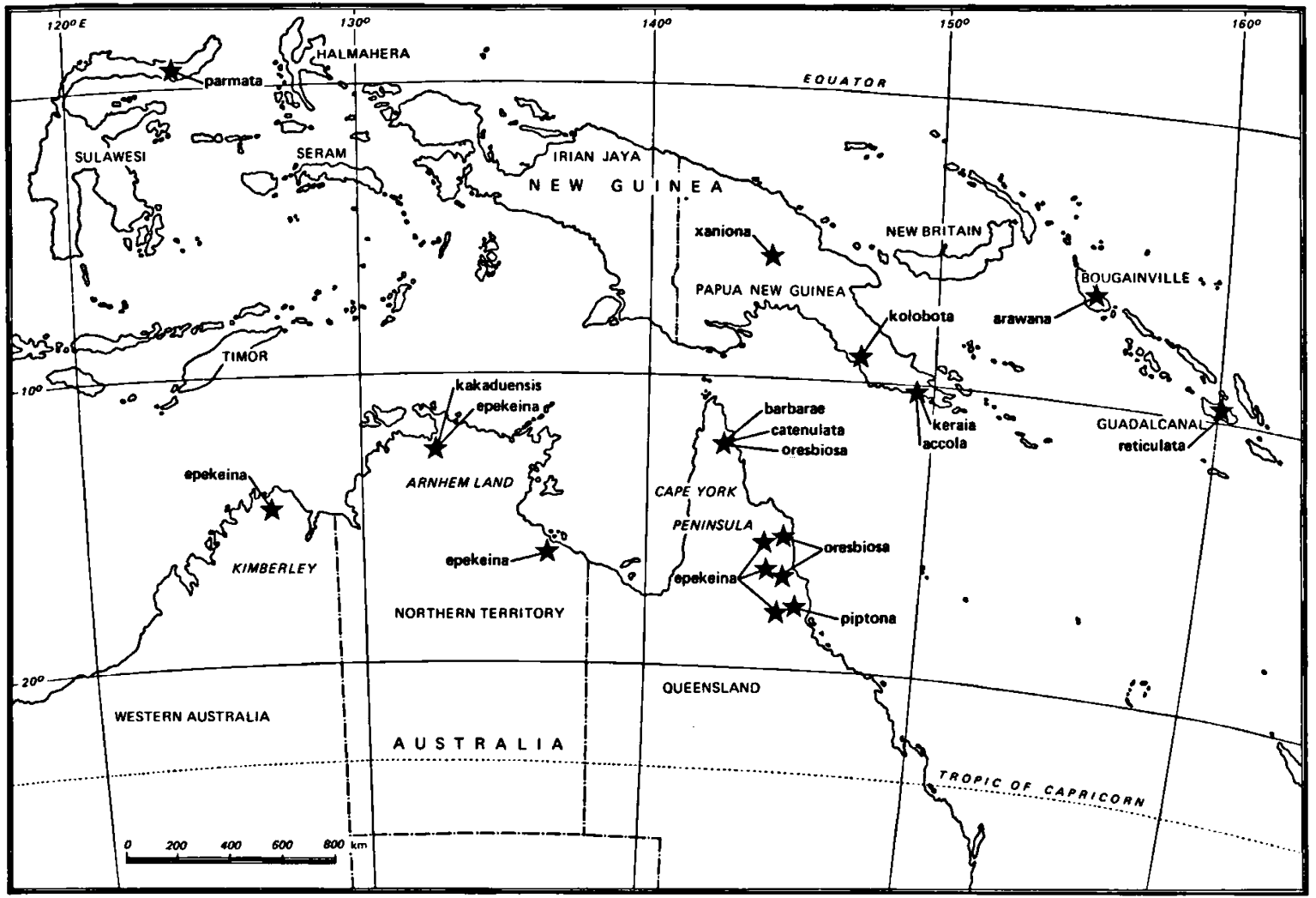

Fig. 1. Map showing distribution of Oecetis reticulata species-group.

(Banks) and $O$. cinarescens (Hagen). A further three species - $O$. bengalica Martynov, $O$. scutulata Martynov and $O$. biramosa Martynov from India, together with $O$. scutata Ulmer from Sumatra, were placed in 'scutata-bengalensis' (sic!) (bengalica) group by Martynov (1936), and another, $O$. orientalis Tsuda (1942) from Japan, in the scutata group by Ulmer (1951). Reticulate patches on tergites 5 to 8 or $\mathbf{6}$ to 8 are known to occur in a number of African species. None of these species, with exception of $O$. testacea, has the distinctive shield-like tergite 8. Kimmins (1957) commented that 'reticulate areas on tergites 5 to 8 links reticulatella Kimmins with choa Mosely and setifera Ulmer', but in africana Kimmins the reticulate areas are only on tergites 6 to 8. Gibbs (1973) united three species - berneri Kimmins, akimi Gibbs and tafo Gibbs from Ghana in 'berneri-group' on the basis of the shape of the clasper ( $=$ inferior appendages) but placed reticulatella in setifera-group and africana in aganda-group. The groupings of these species are rather confused and require close examination in the future.

The thirteen species discussed in this paper, Oecetis testacea from Europe, $O$. lingua Schmid and $O$. fahieni Schmid from Ceylon, are the only ones known to have the distinctive shield-like tergite 8 undivided mid-dorsally and extending over the genital segments. The reticulate patches are present in all species except kakaduensis sp. $\mathrm{n}$. and reticulata; the patches are exceptionally large in accola sp. $\mathrm{n}$. but small in arawana sp. $n$. The Sulawesi species differs in having large patches only on tergite 7 , but none on tergite 6.

The shape of segment 9 in lateral view varies between species and together with distinctive shape of segment 10 and inferior appendages provides clear key characters for species separation which are best appreciated by comparing the figures rather than in a written key.

The species here referred to the reticulata speciesgroup also have characteristic combinations of fore wing venation, vestiture and colour pattern (Fig. 4). 

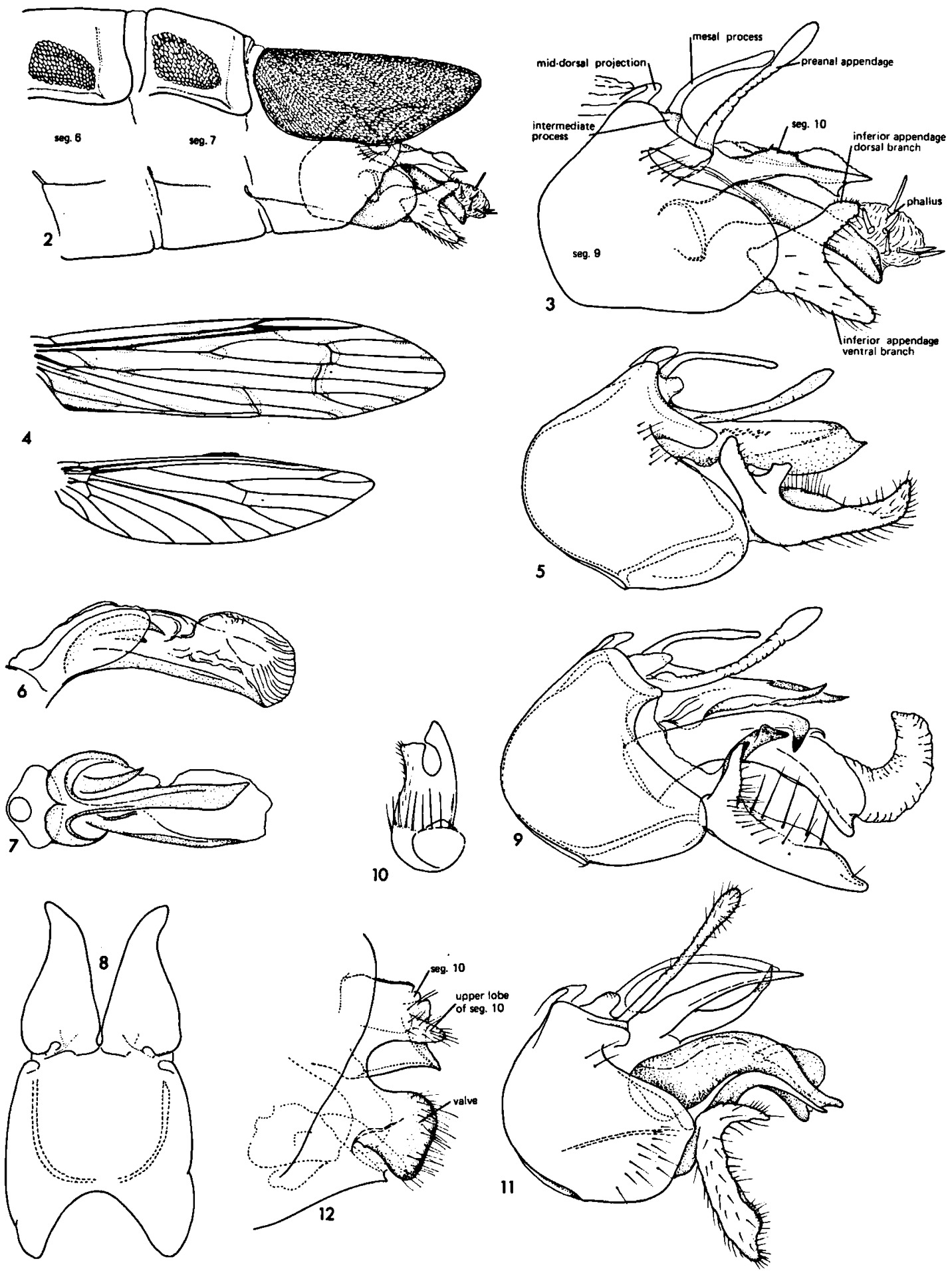

Figs. 2,3. Oecetis catenulata sp.n. 2, Terminal abdominal segments showing position of reticulate patches on tergites 6 and 7 , shield-like tergite 8 and position of male genitalia; 3 , male genitalia lateral.

Figs. 4-8. Oecetis barbarae sp.n. 4, male wing venation; 5, male genitalia lateral; 6, phallus lateral; 7 , phallus ventral; 8 , male genitalia ventral.

Figs. 9,10. Oecetis epekeina sp.n. 9, male genitalia lateral; 10, inferior appendage from distal end.

Figs. 11,12. Oecetis oresbiosa sp.n. 11, male genitalia lateral; 12, female genitalia lateral. 
Fore wing fork 1 sessile, discoidal cell comparatively broad, thyridial cell long, anastomosis almost straight; vestiture with semi-erect hairs mainly along longitudinal veins, darkly shaded areas at anastomoses, at main forks and along oblique cross-veins cu1-cu2-a. Hind wing fork 1 present. Spurs 0:2:2.

The distribution, as known at present, extends over the south-west Pacific area from Sulawesi to New Guinea, Solomon Islands and Northern Australia (Fig. 1).

\section{Depositories}

The following abbreviations given below have been used for the names of the museums and other institutions where specimens are deposited.

ANIC - Australian National Insect Collection, CSIRO, BMNH - British Museum (Natural History), London;

BPBM - Bernice P. Bishop Museum, Honolulu;

MVM - Museum of Victoria, Melbourne;

NTMD - Northern Territory Museum of Art and Sciences, Darwin;

RMNH - Rijksmuseum van Natuurlijke Historie, Leiden;

ZMA - Zoologisch Museum, Universiteit van Amsterdam;

ZMB - Zoological Museum, Bogor.

\section{Oecetis catenulata sp. n. Figs. 2, 3}

Description: Male (Figs. 2,3) abdomen with lateral reticulate patches on tergites 6 and 7 similar in size; segment 9 robust, extended and broadly rounded distally, mid-dorsal projection small; segment 10 consists of a pair of twisted elongate plates with serrate margins; mesal process slightly curved, moderately long, gradually expanded apically. Intermediate processes very short, tuberculate. Preanal appendages slender, dilated apically. Inferior appendages bifid, short, both dorsal and ventral branches robust. Phallus straight, upper distal section membranous, expandable, provided with a number of strong chitinous spines.

Female unknown.

Length of fore wing: $\sigma^{*} 6.8-7.3 \mathrm{~mm}$.

Remarks: Closely resembles $O$. barbarae sp. n. but is recognised by distinct segment 10 and short, robust inferior appendages.

Type material: Holotype $\sigma^{\circ}$, Australia, North Queensland, Cape York Peninsula, Middle Claudie River, Iron Range, 2-9 Oct. 1974, M.S. \& B.J. Moulds (T-9900 MVM). Paratypes: 1 o Gordon Creek, Iron Range, 16 Oct. 1974, M.S. \& B.J. Moulds (genitalia prep. PT-1739 $\sigma$ figured) (MVM); 1 \% same loc., 18 Apr. 1975, M.S. Moulds (MVM); 1 o same loc., 2-9 June 1971, E.F. Riek (ANIC).

Distribution: Australia, Cape York Peninsula; known only from type locality.

Etymology: catenula (Latin), chain, referring to the chain mesh appearance of tergite 8 .

Oecetis barbarae sp. n. Figs. 4-8

Description: Male (Fig. 5) abdominal tergites 6 and 7 with lateral reticulate patches; segment 9 in lateral view broadly rounded, mid-dorsal projection small; segment 10 elongate hood-shaped with wide, asymmetric apical excision, each side terminating in an acute point, mesal process slender, curved at base. Intermediate processes short, truncate. Preanal appendages slender, about as long as segment 10. Inferior appendages with dorsal branch stout, mesal lobe shorter and truncate apically, ventral branch longer, mesal margin expanded, curved dorsally, with row of bristles along the edge, in ventral view elongate triangular (Fig. 8) apices divergent. Phallic structure asymmetric (Figs. 6,7) with one short and one long sclerotized spine arising from bowl-like base, apico-dorsal section membranous.

Female not positively associated.

Length of fore wing: $\sigma^{*} 5.7-6.1 \mathrm{~mm}$. Wing venation (Fig. 4) and colour pattern characteristic of the reticulata species-group.

Remarks: Similar to $O$. catenulata sp. $\mathrm{n}$. it is distinguished by elongate, hood-shaped segment 10 and distally more extended ventral branch of inferior appendages. 
Type material: Holotype $\sigma^{\circ}$, Australia, North Queensland, Iron Range, Gordon Creek $12^{\circ} 46^{\prime} \mathrm{S} 143^{\circ} 16^{\prime} \mathrm{E}, 12$ May 1975 , M.S. \& B.J. Moulds (T-9917 MVM). Paratypes: $1 \sigma^{\circ}$ collected with holotype (genitalia prep. PT-1742 o figured)(MVM); $1 \sigma^{\circ}$ same loc., 18 Apr. 1975, M.S. \& B.J. Moulds (MVM); 1 o' same loc., 28 Apr. 1975, M.S. \& B.J. Moulds (MVM); $1 \sigma$ same loc., 27 Apr. - 4 May 1973, S.R. Monteith (ANIC).

Distribution: Australia, North Queensland, Cape York Peninsula; known only from type locality.

Etymology: Species named after Mrs Barbara J. Moulds in appreciation for her lasting interest in collecting insects on many expeditions to Northern Australia.

\section{Oecetis epekeina sp. n. Figs. 9,10}

Description: Male (Fig. 9) abdominal tergites 6 and 7 with lateral reticulate patches; segment 9 robust, slightly produced distally, anterior margin extended into a short mid-dorsal projection; segment 10 asymmetric, elongate triangular, acutely bifid apically, mesal process about two thirds length. Intermediate processes short, conical. Preanal appendages long, slightly dilated apically. Inferior appendages long and stout; the broad, somewhat flattened dorsal branch apically divided into two lobes (Fig. 10 ), the outer lobe stout, apically truncate, inner lobe slightly longer, hatchet-shaped apically; ventral branch robust, in ventral view sub-triagular, apices rounded. Phallus asymmetric, strongly sclerotized, left upper margin terminates with strong downcurved hook, a small curved hook-like process mid-dorsally, upper distal section membranous, expandable.

Female unknown.

Length of fore wing: $\sigma^{*} 5.4-6.1 \mathrm{~mm}$.

Remarks: Closely resembling $O$. barbarae sp. n. but differing by distinct, acutely terminating segment 10 and larger dorsal branch of inferior appendage.

Type material: Holotype $\sigma^{\circ}$, Australia, North Queensland, $16 \mathrm{~km} \mathrm{~W}$ of Ravenshoe, 2 Jan. 1975, M.S. \& B.J. Moulds (T-9921 MVM). Paratypes: North Queensland, $1 \sigma^{\prime}$ collected with holotype (genitalia prep. PT-1737 ơ figured); 1 o Palmer River, 20 June 1975, E.F. Riek (ANIC); 1 \% McLeod River $15 \mathrm{~km} \mathrm{~W}$ of Mt Carbine, 22-23 June 1975, S.R. Monteith (ANIC); 1 or Kauri Creek, Tinaroo Dam, Atherton Tableland, 24 Apr. 1970, S.R. Curtis (ANIC). Northern Territory, $1 \sigma^{\circ} 9 \mathrm{~km}$ $\mathrm{N}$ by $\mathrm{E}$ of Mudginberri homestead $12^{\circ} 31^{\prime} \mathrm{S} 132^{\circ} 54^{\prime} \mathrm{E}, 10-11$ June 1973, J.C. Cardale (ANIC); 1 o Surprize Creek, $45 \mathrm{~km} \mathrm{SW}$ of Borroloola $16^{\circ} 25^{\prime} \mathrm{S} 136^{\circ} 05^{\prime} \mathrm{E}, 5$ Nov. 1975, J.C. Cardale (ANIC).

Other material examined: Western Australia, $1 \sigma$ Drysdale River (Drysdale Survey Site C5) $14^{\circ} 39^{\prime} \mathrm{S} 126^{\circ} 57^{\prime} \mathrm{E}, 18-21$ Aug 1975, I.F.B. Common \& M.S. Upton (ANIC); 1 \% Carson Escarpment (Site B1) $14^{\circ} 49^{\prime} \mathrm{S} 126^{\circ} 49^{\prime} \mathrm{E}, 9-15$ Aug. 1975, I.F.B. Common \& M.S. Upton (ANIC).

Distribution: Australia, North Queensland, Northern Territory, NW Australia (Kimberleys).

Etymology: epekeina (Greek), on the far side, referring to wide distribution.

Oecetis oresbiosa sp. n. Figs. 11,12

Description: Male (Fig. 11) abdominal tergites 6 and 7 with lateral reticulate patches; segment 9 short and broad, mid-dorsal projection of anterior margin small; segment 10 consists of two sclerotized, curved, apically pointed processes, mesal process gradually curved, almost as long as segment. Intermediate processes short, rounded apically. Preanal appendages slender, slightly clavate distally. Inferior appendages similar to those in epekeina sp. $\mathrm{n}$. but dorsal branch divides from the base into two curved, apically pointed processes of which the outer is the shorter. Phallus strongly curved, distally tapered, apex bluntly rounded.

Female (Fig. 12) abdominal segment 10 with strong, elongate triangular upper lobes, valves truncate, angles rounded.

Length of fore wing: $\sigma^{*} 5.0-5.8 \mathrm{~mm}$; $5.1 \mathrm{~mm}$

Remarks: Closely resembling $O$. epekeina sp. n. but distinguished by absence of curved hook on phallus and dorsal branch of inferior appendage with slender, apically tapering inner process. 
Type material: Holotype $\sigma^{\star}$, Australia, North Queensland, Gordon Creek, Iron Range, 30 Apr. 1975, M.S. \& B.J. Moulds (T-9923 MVM). Paratypes: 1 o' same loc., 27 May 1975, M.S. \& B.J. Moulds (MVM); 1 West Claudie River, Iron Range, 17 Sept. 1974, M.S. \& B.J. Moulds (MVM); 1 o Middle Claudie River, Iron Range, 19 Sept. 1974, M.S. \& B.J. Moulds (genitalia prep. PT-1738 ơ figured)(MVM); 1 ơ same loc., 2-9 Oct. 1974, M.S. \& B.J. Moulds (MVM); 6 o 2 연 Moses Creek, $4 \mathrm{~km} \mathrm{~N}$ by E Mt. Finnigan, $15^{\circ} 47^{\prime} \mathrm{S} 145^{\circ} 17^{\prime} \mathrm{E}$, 14-16 Oct. 1980, J.C. Cardale (genitalia prep PT-1763 \& figured) (ANIC, MVM, ZMA); 1 o Kuranda 360 m, 30 Mar. 1976, W.N.B. Quick (MVM); 4 o Claudie River, Iron Range $12^{\circ} 46^{\prime} \mathrm{S} 143^{\circ} 16^{\prime} \mathrm{E}, 10$ Nov. 1988, K. Walker (MVM).

Distribution: Australia, North Queensland.

Etymology: oresbios (Greek), living in mountains, in reference to the habitat of the species.

Oecetis xaniona sp. n. Figs. 13-15

Description: Male (Fig. 13) abdomen with lateral reticulate patches on tergite 6 and 7 of approximately similar size; segment 9 moderately robust, broad distally, mid-dorsal projection tapered apically; segment 10 bifid distally, mesal process short, robust, slightly curved. Intermediate processes very short, broadly triangular. Preanal appendages slender, dilated apically, longer than segment 10 . Inferior appendages robust, dorsal branch broad, distinctly trifid apically, ventral branch gradually curved, apices divergent laterally (Fig. 15). Phallus (Fig. 14) partially membranous, with strong mesal spine.

Female unknown.

Length of fore wing: $\sigma^{\prime} 7.0 \mathrm{~mm}$.

Remarks: Similar to $O$. barbarae sp. n. but separated from it by broad, distinctly trifid dorsal branch of inferior appendages.

Type material: Holotype $\sigma$, New Guinea, Papua New Guinea, Tari subdistrict, Mt. Hagen alt.1578 m, 21-25 May 1966, W.Vink (genitalia prep. PT-1784 o figured) (RMNH).

Distribution: New Guinea, Papua New Guinea; known from type locality only.
Etymology: derived from xaniona (Greek) comb, referring to the comb-like margin of inferior apendage dorsal branch.

Oecetis piptona sp. n. Figs. 16-18

Description: Male (Figs. 16,17) abdominal segments 6 and 7 with lateral reticulate patches; segment 9 short, distal margin broadly sub-triangular, rounded apically, mid-dorsal projection small; segment 10 in dorsal view broad at base, ventrally with strongly sclerotized bridge connecting both sides, distal section somewhat twisted and tapered to acute apex, mesal process stout, horn-like. Intermediate processes very short, truncate. Preanal appendages moderately long, dilated apically, about as long as segment 10. Inferior appendages short, dorsal branch robust, rounded apically, mesal margin produced into short mesal lobe, ventral branch short. Phallus (Fig. 17) short, stout, curved downward, dorsal sclerotized plate with short upcurved spur.

Female (Fig. 18) abdominal segment 10 with short upper lobes, valves somewhat rectangular, angles rounded.

Length of fore wing: ${ }^{\circ} 6.0 \mathrm{~mm}$; $9.0 \mathrm{~mm}$.

Remarks: Similar to the New Guinea species $\boldsymbol{O}$. kolobota sp. n. and $O$. accola sp. n. but distinguished by different segment 10 and inferior appendages.

Type material: Holotype ơ, Australia, North Queensland, Zillie Falls nr. Millaa Millaa, 22 Feb. 1982, M.S. and B.J. Moulds (T-9934 MVM). Paratypes: 1 o 1 \& collected with holotype (genitalia prep. PT-1735 o*, PT-1765 \& figured) (MVM).

Distribution: Australia, North Queensland; known from type locality only.

Etymology: pipto (Greek), fall, in reference to the habitat.

Oecetis kolobota sp. n. Figs. 19-22

Description: Male (Fig. 19) abdominal segments 6 

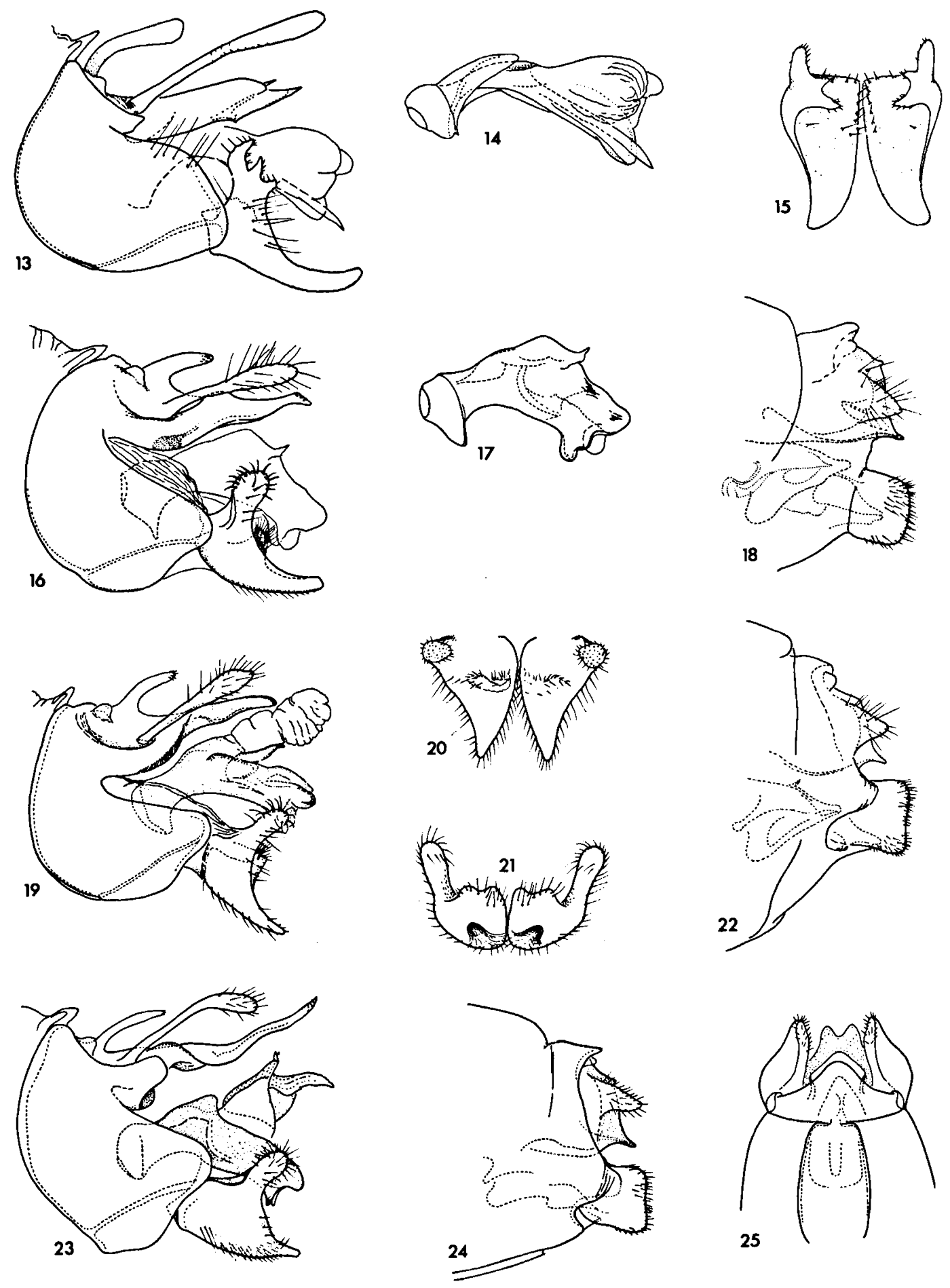

Figs. 13-15. Oecetis xaniona sp.n. 13, male genitalia lateral; 14, phallus lateral; 15, inferior appendages dorsal.

Figs 16-18. Oecetis piptona sp.n. 16, male genitalia lateral; 17, phallus lateral; 18, female genitalia lateral.

Figs. 19-22. Oecetis kolobota sp.n. 19, male genitalia lateral;20, inferior appendages dorsal; 21, inferior appendages from distal end 22, female genitalia lateral.

Figs. 23-25. Oecetis accola sp.n. 23, male genitalia lateral; 24, female genitalia lateral; 25, female genitalia ventral. 
and 7 with lateral reticulate patches of approximately similar size; segment 9 short, distal margin broadly sub-triangular, rounded apically, middorsal projection small; segment 10 digitiform, broad at base, mesal process short. Intermediate processes short, rounded. Preanal appendages about as long as segment 10 , gradually dilated apically. Inferior appendages short, dorsal branch short, rounded apically, mesal margin with bilobed ridge (Fig. 21), ventral branch short, in dorsoventral view triangular (Fig. 20) apices diverging laterally. Phallus with distinct dorsal membranous expansion.

Female (Fig. 22) abdominal segment 10 with upper lobes broadly triangular, valves rectangular, truncate, angles rounded.

Length of fore wing: $\sigma$ 5.3-5.6 mm; $5.0 \mathrm{~mm}$.

Remarks: Similar to the Australian species O. piptona $\mathrm{sp}$. $\mathrm{n}$. but distinguished by different segment 10 and inferior appendages.

Type material: Holotype $\sigma$, New Guinea, Papua New Guinea, Port Moresby, 16 May 1986, A. Wells (T-9968 MVM). Paratypes 2 o 1 \% collected with holotype (genitalia prep. PT-1786

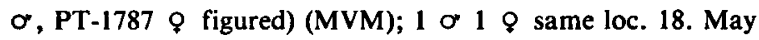
1986, A. Wells (MVM).

Distribution: New Guinea, Papua New Guinea; known only from type locality.

Etymology: derived from kolobos (Greek), shortened, stunted, referring to the short preanal appendages.

Oecetis accola sp. n. Figs. $23-25$

Description: Male (Fig. 23) abdominal tergites 6 and 7 with rather large reticulate patches; segment9 short, distal margin broadly sub-triangular, apically rounded, mid-dorsal projection small, digitiform; segment 10 broad based, slender in distal half, pointed apically; mesal process short, slightly curved. Intermediate processes appear as small rounded tubercles. Preanal appendges moderately long, slender, dilated apically. Inferior appendages short, broad, upper lateral margin extended into apically rounded dorsal branch, ventral branch appears as a short projection. Phallus short, asymmetric with two pointed dorsal lobes.

Female (Figs. 24,25) abdominal segment 10 with upper lobes elongate triangular, valves rectangular, angles rounded, sternite 8 with sclerotized mesoventral plate (Fig. 25).

Length of fore wing: ${ }^{\circ} 6.0 \mathrm{~mm}$; $5.4 \mathrm{~mm}$.

Remarks: similar to $O$. kolobota sp. n. but with slender, apically pointed segment 10 and broader inferior appendages.

Type material: Holotype $\sigma$, New Guinea, Papua New Guinea, Mamai plantation (E of Port Glasgow) $10^{\circ} 16^{\prime} \mathrm{S} 149^{\circ} 30^{\prime} \mathrm{E}$, alt. $150 \mathrm{~m}$, light trap, 5 Feb 1965, R. Straatman (genitalia prep. PT-1781 $\sigma$ figured) (BPBM). Paratypes: $2 \%$ collected with holotype (genitalia prep. PT-1782 $९$ figured) (BPBM).

Distribution: New Guinea, Papua New Guinea; known from type locality only.

Etymology: accola (Latin), neighbour, refers to the existence of another species at the same locality.

Oecetis keraia sp. n. Figs. 26-28

Description: Male (Fig. 26) abdominal tergites 6 and 7 with lateral reticulate patches; tergite 8 elongate, shield-like with honeycomb microstructure similar to other species in reticulata group but a small horn-like lateral projection on each side near distal end (Fig. 27); segment 9 in lateral view almost crescent shaped, mid-dorsal projection digitiform; segment 10 slender, asymmetric, twisted, tapered to pointed apex, mesal process rather small, slender, apical half slightly dilated. Intermediate processes minute. Preanal appendages long, slender, upcurved and dilated apically. Inferior appendages with dorsal branch divided into several short, dorsally and mesally directed branches, ventral branch tapering distally. Phallus broad and strongly sclerotized at base, semi-transparent, apically rounded rod-like projection distally, upper section membranous, expandable, with strong, sclerotized, 

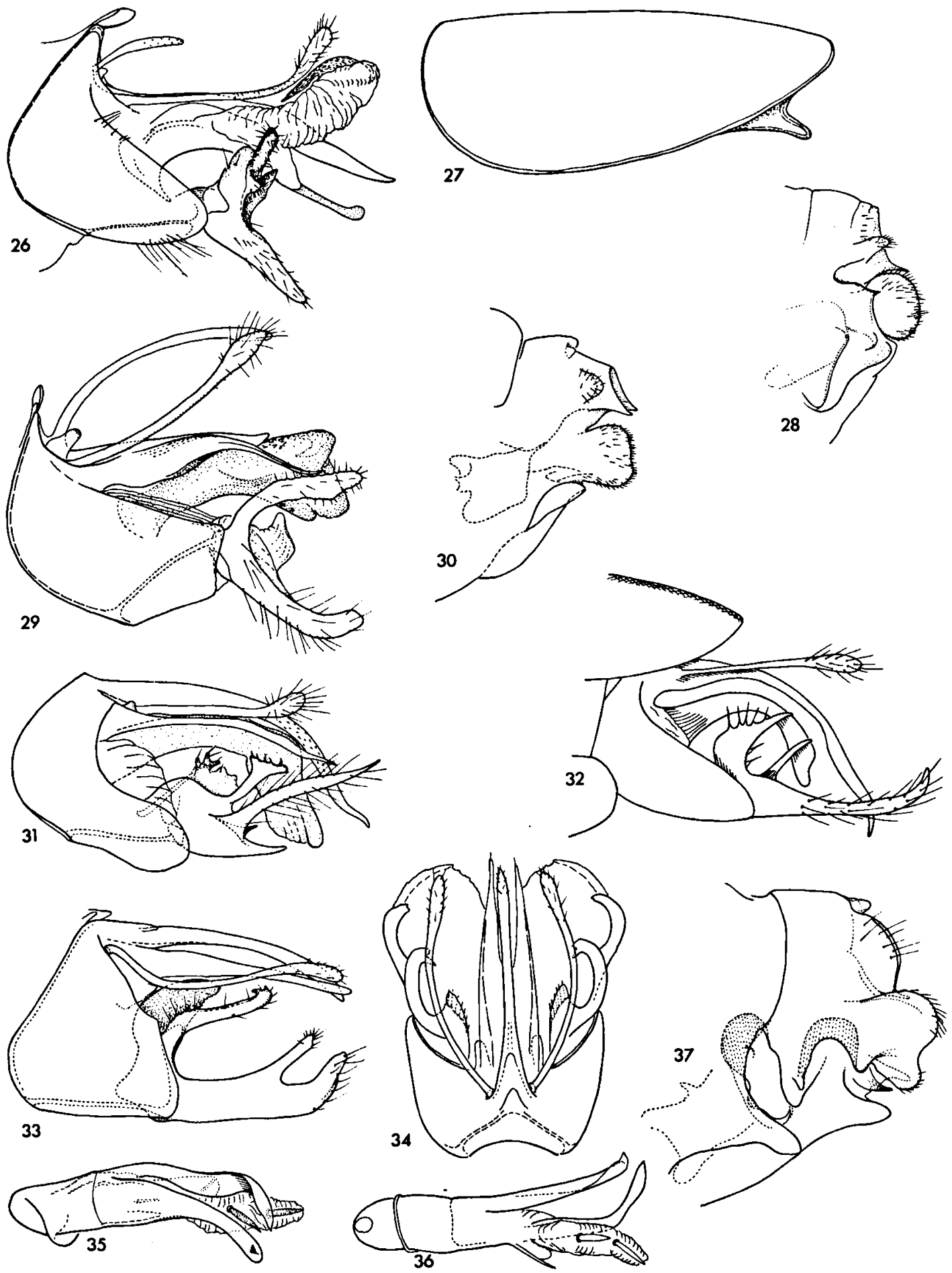

Figs. 26-28. Oecetis keraia sp.n. 26, male genitalia lateral; 27, outline of male tergite 8 lateral; 28, female genitalia lateral.

Figs 29, 30. Oecetis kakaduensis sp.n. 29, male genitalia lateral; 30, female genitalia lateral.

Fig. 31. Oecetis arawana sp.n. male genitalia lateral.

Fig. 32. Oecetis reticulata Kimmins, male genitalia lateral (from Kimmins 1957).

Figs. 33-37. Oecetis parmata sp.n. 33, male genitalia lateral; 34, male genitalia dorsal; 35, phallus lateral; 36, phallus ventral; 37, female genitalia lateral. 
recurved dorsal spine.

Female (Fig. 28) abdominal segment 10 with upper lobe, narrow, rounded apically, valves ovoid.

\section{Length of fore wing: $\sigma^{*} 6.1 \mathrm{~mm}$; $5.5 \mathrm{~mm}$.}

Remarks: Similar to $O$. kakaduensis sp. n., but segment 10 with short slender mesal process, short inferior appendages and characteristic horn-like lateral projection on tergite 8 .

Type material: Holotype $\sigma^{\circ}$, New Guinea, Papua New Guinea, Mamai plantation (E of Port Glasgow) $10^{\circ} 16^{\prime} \mathrm{S} 149^{\circ} 30 \mathrm{E}$, alt. $150 \mathrm{~m}$, light trap, 5 Feb. 1965, R. Straatman (genitalia prep. PT-1732 o figured) (BPBM). Paratype: 1 \% same loc., 7 Feb. 1965, R. Straatman (genitalia prep. PT-1783 \& figured) (BPBM).

Distribution: New Guinea, Papua New Guinea; known from type locality only.

Etymology: keraia (Greek), horn-like projection, in reference to the lateral process on tergite 8 .

Oecetis kakaduensis sp. n. Figs. 29, 30

Description: Male (Fig. 29) abdominal tergites 6 and 7 without lateral reticulated patches; segment 9 with small mid-dorsal projection, broadly rounded anteriorly, extended and angular ventro-distally, reduced dorsally; segment 10 bifurcate, asymmetric, right side branch distinctly shorter, at the base arises a long, slender, slightly curved mesal process. Intermediate processes, short, elongate, apically bluntly rounded. Preanal appendages long, clavate apically. Inferior appendages with dorsal and ventral branches of approximately equal length, mesal margin of ventral branch developed into an angular upward and outward curved lobe. Phallus sclerotized at base, apex membranous, internally with a pair of curved longitudinal sclerites.

Female (Fig. 30) with short rounded upper lobes at the base of segment 10 , valves truncate, apical angles rounded.

Length of fore wing: or $5.7-6.0 \mathrm{~mm} ; \% 5.3-5.9$ $\mathrm{mm}$.
Remarks: Resembling the New Guinea $O$. keraia sp. $n$. but separated by distinct segment 10 and inferior appendages.

Type material: Holotype $\sigma^{\circ}$, Australia, Northern Territory, Nourlangie Camp Lagoon, Kakadu National Park, $12^{\circ} 46^{\prime} \mathrm{S}$ $132^{\circ} 39^{\prime}$ E,4 Sept. 1979, J. Blyth (T-9908 MVM). Paratypes: $10^{\circ}$ 1 \& collected with holotype (MVM); 1 \% 6 \& Nourlangie Creek, $8 \mathrm{~km} \mathrm{~N}$ of Mt Cahill $12^{\circ} 48^{\prime} \mathrm{S} 132^{\circ} 42^{\prime} \mathrm{E}, 16$ June 1973 , J.C. Cardale (genitalia prep. PT-1743 $\sigma^{\circ}$; PT-1744 $\$$ figured) (MVM; ANIC); 1 o 3 \& $12 \mathrm{~km} \mathrm{NNW}$ of Mt Cahill $12^{\circ} 46^{\prime} \mathrm{S}$ $132^{\circ} 39^{\prime} \mathrm{E}, 15-16$ June 1973, J.C. Cardale (ANIC); 2 ? Nourlangie Creek $6 \mathrm{~km}$ E of Mt Cahill $12^{\circ} 52^{\prime} \mathrm{S} 132^{\circ} 46^{\prime} \mathrm{E}, 18$ Nov. 1972, J.C. Cardale (ANIC); 3 ○ Jim Jim Creek on Kakadu highway (blue light trap), 28 May 1988, P. Suter \& A. Wells (MVM, ZMA); 1 o Coonjimba Billabong (blue light trap), 19 May 1988, P. Suter \& A. Wells (MVM).

Distribution: Australia, Northern Territory; Arnhem Land

Etymology: Species name refers to the type locality - Kakadu National Park.

Oecetis arawana sp. n. Fig. 31

Description: Male (Fig. 31) abdominal tergites 6 and 7 each with small indistinct lateral reticulate patches; segment 9 in lateral view crescent shaped, ventral margin produced distally, mid-dorsal projection absent; segment $\mathbf{1 0}$ formed by a pair of asymmetric, slender processes, curved downward and acute apically, mesal process absent. Intermediate processes minute. Preanal appendages long, slender, clavate apically. Inferior appendages with long, slender, apically pointed lateral branch, two ventral branches short, pointed, dorso-lateral branch in lateral view bluntly T-shaped apically, mesal margin irregularly dentate. Phallus curved downward, bluntly rounded apically.

Female unknown.

Length of fore wing: $0^{\circ} 4.5 \mathrm{~mm}$.

Remarks: Closely resembles $O$. reticulata but is separated by different inferior appendages. 
Type material: Holotype ơ, New Guinea, Papua New Guinea, Bougainville Island, Arawa $06^{\circ} 10^{\prime} \mathrm{S} 157^{\circ} 15^{\prime} \mathrm{E}, 12$ Mar. 1988, C. Yule (genitalia prep. PT-1741 o॰ figured) (T-9899 MVM).

Distribution: Bougainville Island, Papua New Guinea; known from type locality only.

Etymology: Species name refers to the type locality.

Oecetis reticulata Kimmins, 1957. Fig. 32

Oecetis reticulata Kimmins, 1957: 302.

According to the original description there are no lateral reticulate patches on tergites 6 and 7 and the figures do not indicate the presence of the middorsal projection of segment 9 or the intermediate processes at the base of segment 10 . The species is similar to $O$. arawana sp. n. from Bougainville but differs in slender calliper-like inferior appendages.

Female unknown.

Length of fore wing: $\sigma \times 4.5 \mathrm{~mm}$

Type material: Holotype $\sigma^{\prime}$, Guadalcanal, Honiara, $09^{\circ} 26^{\prime} \mathrm{S}$ $159^{\circ} 57^{\prime}$ E, 4-8 Oct. 1953, J.D. Bradly (BMNH) (not seen).

No new material has been available for study.

Distribution: Guadalcanal; known from type locality only.

Oecetis parmata sp. n. Figs. 33-37

Description: Male, the reticulated shield-like tergite 8 has rather thick honeycomb structure; reticulation on tergite 6 absent, but the reticulate patches on tergite 7 rather extensive and almost meet dorsally; segment 9 in lateral view (Fig. 33) subtriangular, mid-dorsal projection small, directed distally; segment 10 (Fig. 34) consists of an upper mesal process and two slightly longer, asymmetric lateral processes. Preanal appendages long and slender, slightly clavate apically. Inferior appendages robust, with curved dorsal branch and robust palm-like ventral branch with a lateral upcurved digitiform process. Phallus (Figs. 35, 36) asymmetric, apical section membranous with several strong, distally directed chitinous spines.

Females (Fig. 37) abdominal segment 8 disto-lateral angle extended into apically tapering process, ventral margin of segment 9 forms two pockets which in copulation most likely act as receptacles to the upcurved digitiform processes of the male inferior appendages; the valves are large, indented distally, lower angle enlarged, upper lobes at the base of segment 10 absent.

Length of fore wing: $\circ$ 4.4-5.1 mm; $\$$ 4.5 - 4.7 $\mathrm{mm}$.

Remarks: Closest in general appearance to $O$. reticulata but differs by distinct genitalic structures.

Type material: Holotype ơ, Sulawesi, Dumoga-Bone National Park, Toraut-Tumpah River junction $00^{\circ} 34^{\prime} \mathrm{N} 123^{\circ} 55^{\prime} \mathrm{E}$, light trap, 16 May 1985, A. Wells (T-9903 MVM). Paratypes: $10^{\circ}$ Edwards Camp, alt. 650m, 22 May 1985, A. Wells (genitalia prep. PT-1515 o figured) (MVM); 3 ơ 2 \% Toraut- Tumpah River junction, at light, Aug. 1985, D. Dudgeon (BMNH, ZMA, ZMB);

1 o Base Camp, River Toraut, alt. $211 \mathrm{~m}$, MV-light, 4 Oct - 8 Nov. 1985, M. Malipatil (NTMD); 2 ९ Pononontuma River bridge $6.5 \mathrm{~km} \mathrm{~N}$ of Malibagu $00^{\circ} 25^{\prime} \mathrm{N} 123^{\circ} 58^{\prime} \mathrm{E}, 24$ May 1985, A. Wells \& M. Wilson (genitalia prep. PT-1761\& figured) (MVM); 1 \& Motolanga River, Dolodua- Malibagu road $00^{\circ} 28^{\prime} \mathrm{N} 123^{\circ} 58^{\prime} \mathrm{E}$, at light, 7 May 1985, A. Wells (MVM).

Distribution: Northern Sulawesi (Sulawesi Utara)

Etymology: From parmata (Latin), armed with a shield, in reference to the shield-like tergite 8 .

\section{Acknowledgements}

I am indebted to Mr and Mrs Moulds of Sydney for presenting extensive caddis-fly material, particularly from Cape York peninsula; to Dr Alice Wells of the Zoology Department, University of Adelaide for the specimens from Sulawesi; to Ms J.C. Cardale of Australian National Insect Collection, Canberra, Ms C. Yule of Bougainville and to Dr G.M. Nishida, Department of Entomology, Bishop Museum, Honolulu for the specimens which made this study possible.

This paper has been registered as Results of Project Wallace No 81; the project was sponsored by the Royal Entomological Society of London and the Indonesian Institute of Science. 


\section{References}

Betten, C., 1934. The caddis-flies or Trichoptera of New York State. New York State Mus. Bull., 292: 1-576.

Gibbs, D.G., 1973. The Trichoptera of Ghana. Deutsche ent. Zs., (nF) 20: 363-424.

Kimmins, D.E., 1957. Neuroptera and Trichoptera collected by Mr J.D. Bradley on Guadalcanal Island 1953-54. Bull. Brit. Mus. (nat.Hist.), (Ent.) 5: 287-308.

McLachlan, R., 1877. A monographic revision and synopsis of the Trichoptera of the European fauna, 6: 281-348 (J. van Voorst, London).

Martynov, A.V., 1936. On a collection of Trichoptera from the Indian Museum, 2.Integripalpia. Rec. Ind. Mus., 38: 239-306.
Ris, F., 1904. Ein unbekanntes Organ der Phryganiden Oecetis notata und Oecetis testacea. Vierteljahrsschr. naturforsch. Ges.Zürich, 49: 370-374, figs 5-6, pl. 12.

Ris, F., 1905. Über ein unbekanntes Organ der Phryganiden Oecetis notata und Oecetis testacea. Mitt. Schweiz. ent. Ges., 11: 63-65.

Ross, H.H., 1944. The Caddis Flies, or Trichoptera, of Illinois. Bull. Ill. nat. Hist. Surv., 23: 1-326.

Tsuda, M., 1942. Japanische Trichopteren. I. Systematik. Mem. Coll. Sci., Kyoto Imp. Univ., (B) 17: 239-339.

Ulmer, G., 1951. Köcherfliegen (Trichopteren) von den SundaInseln (1) Arch. Hydrobiol., Suppl. 19: 1-528.

Received: 6 June 1989

Revised: 8 August 1989 